\title{
Mathematical Model of the Transmission Dynamics of Novel Corona Virus (COVID-19) Pandamic Disease with Optimal Control
}

\author{
${ }^{1}$ Getachew Beyecha Batu, ${ }^{2}$ Eshetu Dadi Gurmu \\ ${ }^{1}$ Department of Mathematics, Samara University, Samara, Ethiopia \\ ${ }^{2}$ Department of Mathematics, Wollega University, Nekemte, Ethiopia \\ 1'gechobeyecha143@gmail.com, 2eshetudadi1@gmail.com
}

Received: July 30, 2021. Revised: November 5, 2021. Accepted: November 23, 2021. Published: December 7, 2021.

\begin{abstract}
In this paper, we have developed a deterministic mathematical model that discribe the transmission dynamics of novel corona virus with prevention control. The disease free and endemic equilibrium point of the model were calculated and its stability analysis were prformed. The reproduction number $\mathrm{RO}$ of the model which determine the persistence of the disease or not was calculated by using next generation matrix and also used to determine the stability of the disease free and endemic equilibrium points which exists conditionally. Furthermore, sensitivity analysis of
\end{abstract}

the model was performed on the parameters in the equation of reproduction to determine their relative significance on the transmission dynamics of COVID- 19 pandemic disease. Finally the simulations were carried out using MATLAB R2015b with ode45 solver. The simulation results illustrated that applying prevention control can successfully reduces the transmission dynamic of COVID-19 infectious disease.

Keywords: Analysis, Control, COVID-19, Reproducction Number, Transmission dynamic . 


\section{INTRODUCTION}

Covid-19 is an infectious disease caused by the newly-discovered virus strain SARS-CoV-2 and spreads through the respiratory trac [19]. Coronavirus disease is an infectious disease caused by recently discovered COVID-19 virus. It is a new strain that was discovered in 2019 and has not been yet identified in humans[7, 8, 10]. The COVID-19 is a novel coronavirus that was first reported to the world health organization office in China on 31 December 2019 [5]. On January 30, 2020, the epidemic was declared a health emergency of international concern. On February 2020, world healthy organization announced a name for the new coronavirus-19 disease "COVID-19" [8]. Most of the studies about COVID-19 suggest that coronaviruses, including preliminary information, COVID-19 virus can stay on the surface of an object for hours or days[7]. The most common symptoms of COVID-19 are fever, dry cough, fatigue, tiredness, shortness of breath and breathing difficulties [6, 9]. Some of the patients may have aches and pains, nasal congestion, running noses, a sore throat or diarrhea. The Symptoms are usually mild, but they may get worse. When an infected person coughs or sneezes, it spreads from person to person through droplets in the air and between people who are in close contact with one another with in about half dozen feet. Most of the transmission is happening through respiratory droplets that we may inhale from close contact with one another [3]. Recent studies have evaluated the survival of the COVID19 virus on various surfaces and reported how long the virus can survive up to 72 hours on plastic and stainless steel, up to four hours on copper, and up to 24 hours on cardboard. Apr 7, 2020. The incubation period within which the symptoms would appear is
$2-14$ days[9]. In the most severe cases infection can cause pneumonia, sever acute respiratory syndrome and even death. There is no specific treatment for the disease caused by COVID-19 infectious viruse. However, many of the symptoms can be treated and therefore the treatment is based on the patient's clinical condition. The best ways that are recommended by WHO to prevent the novel coronavirus (COVID-19) are, taking vaccine of covid-19, washing hands often with soap and water, if not available use hand sanitizer, avoid touching your eyes, nose and avoid close contact with others, cover your mouth/nose with a tissue or sleeve when coughing or sneezing and so on [8].

At the moment, COVID-19 is of great emphasis to researchers, governments, and all people because of the high rate of the infection outbreak and the significant number of deaths that occurred. Chen et al [11] developed Bats-HostsReservoir -People transmission network model for simulating the potential spread of infection from the source of infection (possibly from bats) to humans infection. Bats-Hosts-Reservoir's network has been difficult to study clearly, and the public concerns have been focused on streaming media from Huanan Seafood Wholesale Market (reservoir) to people, they simplified the model as Reservoir-People (RP) transmission network model. The model showed that the transmission of SARS-CoV-2 was higher than the Middle Eastern countries are similar to severe acute respiratory syndrome, but lower than South Korea's MERS. Furthermore, Chayu Yang and Jin Wang [13] model describes the multiple transmission pathways in the infection dynamics, and emphasize the role of environmental hosts in the spread of this disease. Analysis and numerical results show that coronavirus infections will continue to be prevalent, requiring long-term disease 
prevention and intervention plans. In this paper, we propose a mathematical model for pandemic Covid-19 transmission dynamics, which accounts for optimal control. We consider a special class called quarantine class at which an inividuals are compulsory quarantine to minimize the expansion of COVID-19 and get treatment at this class . To join this class, individuals must present evidences to be exposed, infected in symptomatic and asymptomatic phase. Evidences could be the measures put in place to ensure an individual with risk living environment. We believe that investing in this group of individuals could significantly curtail the spread of COVID-19 while ensuring adding an optimal control to the model reduce the dynamic transmission of the disease.

\section{MODEL ASSUMPTION AND FORMULATION}

The individuals that make up the population can be divided into different classes. The population function in the compartment is the timedifferentiable $(t)$, and the output of the model is fully determined by the parameter values and the initial conditions which is deterministic. The COVID-19 model sub-divided the total population $N(t)$ at any time $t$, into five different compartments, namely,

- Susceptible individuals ( vulnerable or prone to the disease) $S(t)$.

- Exposed individuals ( already infected but are not yet infectious ) $E(t)$.

- Asymptomatic individuals ( both infected and infectious but do not show any symptoms of the disease) $A(t)$.

- Infected individuals ( infectious and shows a symptoms of the disease ) $I(t)$ and
- Quarantine individuals(infectious and compulsory quarantine to minimize the expansion of COVID-19) $Q(t)$.

Then the total number of population $N(t)$ in the model at time $t$, is given by

$$
N(t)=S(t)+E(t)+A(t)+I(t)+Q(t) .
$$

The model formed assumed that; susceptible individuals are recruited (by birth or immigration) into the population at a constant rate $\pi$. A proportion of these susceptible individuals become exposed to COVID-19 infection at a rate $\lambda$ when their is an effective contact with an infectious individuals at a rate $\beta$ and move to $E(t)$ class that may lead to infectious class or quarantine class due to reduce the expansion of the virus at a rate $\varphi$. The force of infection(or rate of infection) of the model is given by $\lambda=\frac{\beta(I+q A)}{N}$, where $\beta$ is an effective contact rate and $q$ is the transmission coefficient for the asymptomatic individuals. If $q>1$ then the asymptomatic individuals infect the susceptible indiviuals more likely than the infective individuals. If $q<1$, then the infected individuals have a good chance to infect the susceptible individuals than asymptomatic individuals and if $q=1$, then both asymptomatic and infected individuals have equal chance to infect the susceptible individuals. After the disease incubation priod $\frac{1}{\eta}$, where $\eta$ is a per capita rate of becoming infections a proportion of $p$ of the individuals in $E(t)$ may develop a symptoms of the COVID-19 infection and move to the infected compartment $I(t)$ at a rate $p \eta$ and the rest become asymptomatic individuals with COVID-19 infection with probability $(1-p)$ and move to the $A(t)$ at a rate $(1-p) \eta$. Furthermore, an individual in asymptomatic $A(t)$ compartment move to the quarantine class $Q(t)$ at a rate $\gamma$ due to they distrust that they are exposed to COVID-19 infctious disease. The infected individuals $I(t)$ move 
to quarantine class $Q(t)$ and get a treatement at a rate $\alpha$. A quarantine individuals may revert to the susceptible class $S(t)$ after gettin treatment and lossing their immunity at a rate $\theta$. Both infectious individuals in $N(t)$ have induced death at a rate $\zeta$. All individuals in $N(t)$ suffers natural mortality at a rate $\mu$ and all the parameter in the model are non-negative.

Depending on the basic assumptions together with the both model variables and parameters, the schematic diagram of the flow between the five compartments of the modified model can be given as in figure as follow.

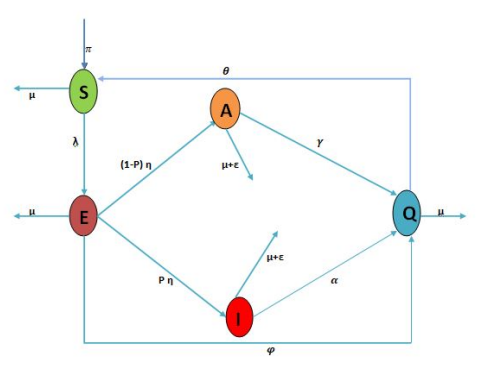

Figure 1: Schematic diagram of the transmission dynamic of COVID-19 model

Based on the model assumptions, notations of the variables and parameters the model equations are formulated into a system of five nonlinear
ODEs as follows:

$$
\left\{\begin{array}{l}
\frac{d S}{d t}=\pi+\theta Q-(\lambda+\mu) S, \\
\frac{d E}{d t}=\lambda S-(\mu+\eta+\varphi) E, \\
\frac{d A}{d t}=(1-p) \eta E-(\mu+\gamma+\zeta) A, \\
\frac{d I}{d t}=p \eta E-(\mu+\alpha+\zeta) I, \\
\frac{d Q}{d t}=\alpha I+\gamma A+\varphi E-(\theta+\mu) Q .
\end{array}\right.
$$

With non-negative initial conditions denoted by $S(0)=S_{0}>0, E(0)=E_{0} \geq 0, A(0)=A_{0} \geq$ $0, I(0)=I_{0} \geq 0, Q(0)=Q_{0} \geq 0$.

\section{MODEL ANALYSIS}

\section{A. Invariant Region}

In this section, we deal about an invariant region in which the model solutions of the system are uniformly bounded, which is a proper subset of $\Omega \subset R_{+}^{5}$.

The total population $N$ of the model at any time $t$ is given by

$$
\begin{gathered}
N(t)=S(t)+E(t)+A(t)+I(t)+Q(t) \\
\frac{d N}{d t}=\frac{d S(t)}{d t}+\frac{d E(t)}{d t}+\frac{d A(t)}{d t}+\frac{d I(t)}{d t}+\frac{d Q(t)}{d t} \\
\frac{d N}{d t}=\pi-\mu N-\zeta(A+I) .
\end{gathered}
$$




$$
\frac{d N}{d t} \leq \pi-\mu N .
$$

By solving equation 3.2 we obtain $0 \leq N \leq \frac{\pi}{\mu}$. Therefore, the feasible solution set of the system equation 2.1 of the model remain in the region:

$$
\Omega=\left\{(S, E, A, I, Q) \in \mathbb{R}_{+}^{5}: N \leq \frac{\pi}{\mu}\right\} .
$$

\section{B. Existence of the model solutions.}

The reliability of any mathematical model depends on whether the given equations have solutions, and if the solution exists then it is unique. We shall use the Lipschitz condition to verify the existence and uniqueness of solution for the system of equation 2.1.

Theorem 1. : Let $\Omega$ denote the region $0<\alpha \leq$ $\mathbb{R}$. Then the model equations (2.1) together with the initial conditions $S(0)>0, E(0) \geq 0$, $A(0) \geq 0, I(0) \geq 0, Q(0) \geq 0$ exist in $\mathbb{R}_{+}^{5}$.

proof: Let the right hand side of the system of equation (2.1) can be expressed as follows:

$$
\left\{\begin{array}{l}
f_{1}(S, E, A, I, Q)=\pi+\theta Q-(\lambda+\mu) S \\
f_{2}(S, E, A, I, Q)=\lambda S-(\mu+\eta+\varphi) E \\
f_{3}(S, E, A, I, Q)=(1-p) \eta E-(\mu+\gamma+\zeta) A \\
f_{4}(S, E, A, I, Q)=p \eta E-(\mu+\alpha+\zeta) I \\
f_{5}(S, E, A, I, Q)=\alpha I+\gamma A+\varphi E-(\theta+\mu) Q .
\end{array}\right.
$$

We have to show that $\frac{\partial f_{i}}{\partial x_{j}}, i, j=1,2,3,4,5$ are continues and bounded in $\Omega$.

According to [1], let $\Omega$ denote the region $\Omega=(S, E, A, I, Q) \in \mathbb{R}_{+}^{5} ; N \leq \frac{\pi}{\mu}$.

Then equations (2.1) have a unique solution if $\frac{\partial f_{i}}{\partial x_{j}}, i, j=1,2,3,4,5$ are continuous and bounded in $\Omega$.

Here, $x_{1}=S, x_{2}=E, x_{3}=A, x_{4}=I, x_{5}=Q$.

\begin{tabular}{|c|}
\hline $\begin{array}{l}\text { For } f_{1} \text { : } \\
\left|\frac{\partial f_{1}}{\partial S}\right|=|-(\lambda+\mu)|<\infty \\
\left|\frac{\partial f_{1}}{\partial E}\right|=0<\infty \\
\left|\frac{\partial f_{1}}{\partial A}\right|=\left|-\frac{\beta q}{N}\right|<\infty \\
\left|\frac{\partial f_{1}}{\partial I}\right|=\left|\frac{-\beta}{N}\right|<\infty \\
\left|\frac{\partial f_{1}}{\partial Q}\right|=|\theta|<\infty\end{array}$ \\
\hline $\begin{array}{l}\text { For } f_{2} \text { : } \\
\left|\frac{\partial f_{2}}{\partial S}\right|=|\lambda|<\infty, \\
\left|\frac{\partial f_{2}}{\partial E}\right|=|-(\eta+\varphi+\mu)|<\infty, \\
\left|\frac{\partial f_{2}}{\partial A}\right|=\left|\frac{\beta q}{N}\right|<\infty, \\
\left|\frac{\partial f_{2}}{\partial I}\right|=\left|\frac{\beta}{N}\right|<\infty, \\
\left|\frac{\partial f_{2}}{\partial Q}\right|=0<\infty .\end{array}$ \\
\hline $\begin{array}{l}\text { For } f_{3} \text { : } \\
\left|\frac{\partial f_{3}}{\partial S}\right|=0<\infty \\
\left|\frac{\partial f_{3}}{\partial E}\right|=|(1-p) \eta|<\infty \\
\left|\frac{\partial f_{3}}{\partial A}\right|=|-(\gamma+\mu+\zeta)|<\infty \\
\left|\frac{\partial f_{3}}{\partial I}\right|=0<\infty \\
\left|\frac{\partial f_{3}}{\partial Q}\right|=0<\infty\end{array}$ \\
\hline $\begin{array}{l}\text { For } f_{4} \text { : } \\
\left|\frac{\partial f_{4}}{\partial S}\right|=0<\infty, \\
\left|\frac{\partial f_{4}}{\partial E}\right|=|p \eta|<\infty, \\
\left|\frac{\partial f_{4}}{\partial A}\right|=|\phi|<\infty, \\
\left|\frac{\partial f_{4}}{\partial I}\right|=|-(\alpha+\mu+\zeta)|<\infty, \\
\left|\frac{\partial f_{4}}{\partial Q}\right|=0<\infty .\end{array}$ \\
\hline $\begin{array}{l}\text { For } f_{5}: \\
\left|\frac{\partial f_{5}}{\partial S}\right|=0<\infty \\
\left|\frac{\partial f_{5}}{\partial E}\right|=|\varphi|<\infty \\
\left|\frac{\partial f_{5}}{\partial A}\right|=|\gamma|<\infty \\
\left|\frac{\partial f_{5}}{\partial I}\right|=|\alpha|<\infty \\
\left|\frac{\partial f_{5}}{\partial Q}\right|=|-(\theta+\mu)|<\infty\end{array}$ \\
\hline
\end{tabular}

The continuity and the boundedness are verified here under:
Thus, all the partial derivatives $\frac{\partial f_{i}}{\partial x_{j}}, i, j=$ $1,2,3,4,5$ exist, continuous and bounded in $\Omega$. Hence, by Derrick and Groosman theorem, a solution for the model (2.1) exists.

\section{Positivity of the Solution of the Model}

It is also necessary to prove that all the model variables of of equation 2.1 are non-negative; 
Such that the solution of the system with nonnegative initial conditions remains positive for all $t>0$. The following lemma shows about this facts.

Lemma 1. (Positivity) Solutions of the model equations 2.1 together with the initial conditions $S(0)>0, E(0) \geq 0, A(0) \geq 0, I(0) \geq$ $0, Q(0) \geq 0$ are always positive $(O R)$ the model variables $S(t), E(t), A(t), I(t)$ and $Q(t)$ are positive for all t and will remain in $\mathbb{R}_{+}^{5}$.

Proof: Positivity of the model variables are shown separately for each of the model variables $S(t), E(t), A(t), I(t)$ and $Q(t)$.

Positivity of $\boldsymbol{P}(\boldsymbol{t})$ : The model equation in 2.1) given by $\frac{d S}{d t}=\pi+\theta R-(\mu+\lambda) S$ can be expressed without loss of generality (WOLG), after eliminating the terms $(\pi+\theta R)$ which are appearing on the right hand side, as an inequality as $\frac{d S}{d t} \geq-(\mu+\lambda) S$. Solving the above problems with the method of separable variables and on applying integration, the solution of the foregoing differentially inequality can be obtained as $S(t) \geq e^{-\mu t-\frac{\beta}{N} \int(I+q A) d t}$. Remember that regardless of the sign of the exponent, the exponential function is always non-negative.

Thus, the exponential function $e^{-\mu t-\frac{\beta}{N} \int(I+q A) d t}$ is a non-negative quantity.

Hence, it can be concluded that $S(t)>0$.

Similarly, solving the system of differential equation of the model, we obtain the exponential function:

$$
\begin{aligned}
& E(t) \geq e^{-(\mu+\eta+\varphi) t}, \\
& A(t) \geq e^{-(\mu+\gamma+\zeta) t}, \\
& I(t) \geq e^{-(\mu+\alpha+\zeta) t}, \\
& Q(t) \geq e^{-(\mu+\theta) t} .
\end{aligned}
$$

Remember that regardless of the sign of the exponent, the exponential function is always nonnegative. Hence, it can be concluded that all the solutions of model equations are positive.
Thus, the model variables $S(t), E(t), A(t), I(t)$ and $Q(t)$ representing population sizes of various types of cells are non-negative quantities and will remain in $\mathbb{R}_{+}^{5}$ for all $t$.

\section{Disease Free Equilibrium Points}

In this part, we got the balance point at which the epidemic of residents was eliminated. Setting the right-hand side of Equation 2.1 to zero and letting

$$
\begin{gathered}
E=A=I=Q=0, \quad \text { leads to } \\
E_{0}=\left\{S^{0}, E^{0}, A^{0}, I^{0}, Q^{0}\right\}=\left\{\frac{\pi}{\mu}, 0,0,0,0\right\}
\end{gathered}
$$

\section{( . 7KH\%DMFI5 HLRGXFURQ1 XP EHU}

The basic reproduction rate, expressed as $R_{0}$, is the average number of secondary infections caused by an infectious individual during his or her entire period of infectiousness [1]. The basic reproduction number is an important dimensionless quantity in epidemiology because it has a threshold in the study of a disease both for predicting its outbreak and for evaluating its control strategies [1]. Therefore, whether disease persists or disappears in the community depends on the values of the reproduction number, $R_{0}$. Furthermore, the stability of the equilibrium point can be analyzed using $R_{0}$ [2]. If $R_{0}<1$ it means that every infectious individual will cause less than one secondary infection which is impossible and hence the disease will die out and when $R_{0}>1$ every infectious individual will cause more than one secondary infection and hence the disease will invade the population. It is obtained by taking the largest (dominant) eigenvalue (spectral radius).

$$
R_{0}=\left[\frac{\partial F_{i}\left(x_{0}\right)}{\partial x_{j}}\right]\left[\frac{\partial V_{i}\left(x_{0}\right)}{\partial x_{j}}\right]^{-1}
$$

where $F_{i}$ be the rate of appearance of new criminal in compartments, $V_{i}$ is the transfer of individuals out of the compartment by another means, 
$x_{0}$ is the disease free equilibrium point. We can find the basic reproduction number using the next generation matrix approch [1, 2].

Thus the associated matrices $\mathrm{F}$ and $\mathrm{V}$ for the new infectious terms and the remaining transition terms are respectively given by:

$$
\begin{gathered}
F_{i}=\left[\begin{array}{c}
\frac{\beta(I+q A) S}{N} \\
0 \\
0 \\
0
\end{array}\right] \text { and } \\
V_{i}=\left[\begin{array}{c}
(\eta+\varphi+\mu) E \\
-(1-p) \eta E+(\mu+\gamma+\zeta) A \\
-p \eta E+(\alpha+\mu+\zeta) I \\
-\varphi E-\gamma A-\alpha I+(\theta+\mu) Q
\end{array}\right]
\end{gathered}
$$

The Jacobian of $F_{i}$ and $V_{i}$ at the disease free equilibrium point $E_{0}$ takes the form respectively as:

$$
\begin{aligned}
F\left(E_{0}\right) & =\left[\begin{array}{ccc}
0 & \beta q & \beta \\
0 & 0 & 0 \\
0 & 0 & 0
\end{array}\right] \text { and } \\
V\left(E_{0}\right) & =\left[\begin{array}{cccc}
b & 0 & 0 & 0 \\
-(1-p) \eta & c & 0 & 0 \\
-p \eta & 0 & d & 0 \\
-\varphi & -\gamma & -\alpha & e
\end{array}\right]
\end{aligned}
$$

where, $b=\eta+\varphi+\mu, \quad c=\mu+\gamma+\zeta$,

$d=\alpha+\mu+\zeta, \quad e=\theta+\mu$.

It can be verified that the matrix $V\left(E_{0}\right)$ is nonsingular as its determinant is non-zero.

That is

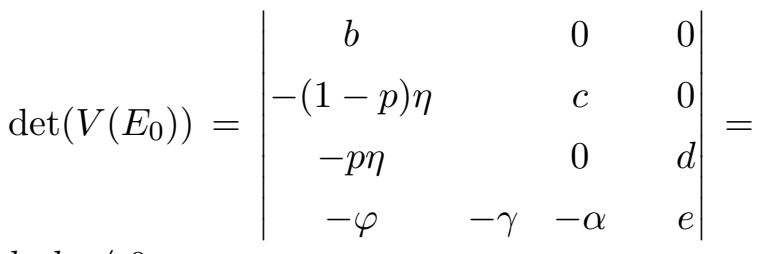

$b c d e \neq 0$

Since $\operatorname{det}\left(V\left(E_{0}\right)\right) \neq 0$ then $V\left(E_{0}\right)$ is invertable and the inverse is given by .

$$
\left(V\left(E_{0}\right)\right)^{-1}=\frac{\operatorname{Adj}(V)}{\operatorname{det}(V)}
$$

Then after some algebraic computations the inverse matrix is constructed as follows:

$$
\left[V\left(E_{0}\right)\right]^{-1}=\left[\begin{array}{cccc}
\frac{1}{b} & 0 & 0 & 0 \\
\frac{(1-p) \eta}{b c} & \frac{1}{c} & 0 & 0 \\
\frac{p \eta}{b d} & 0 & \frac{1}{d} & 0 \\
\frac{c p \eta \alpha+c d \varphi+d \gamma \eta(1-p)}{b c d e} & \frac{\gamma}{c e} & \frac{\alpha}{d e} & \frac{1}{e}
\end{array}\right]
$$

Now,

$$
\left[F\left(E_{0}\right)\right]\left[V\left(E_{0}\right)\right]^{-1}=\left[\begin{array}{cccc}
\frac{\beta \eta(p q c+(1-p) d)}{b c d} & \frac{\beta}{c} & \frac{\beta q}{d} & 0 \\
0 & 0 & 0 & 0 \\
0 & 0 & 0 & 0 \\
0 & 0 & 0 & 0
\end{array}\right]
$$

Thus the eigenvalues of the matrix (3.6) are:

$\lambda_{1}=\frac{\beta \eta(p q c+(1-p) d)}{b c d}, \lambda_{2}=0, \quad \lambda_{3}=0, \quad \lambda_{4}=0$ where, $b=\eta+\mu+\varphi, \quad c=\mu+\gamma+\zeta, \quad d=$ $\alpha+\mu+\zeta, \quad e=\mu+\theta$

Now, $\left[F\left(E_{0}\right)\right]\left[V\left(E_{0}\right)\right]^{-1}$ is the next-generation matrix of system 2.1). It follows that the spectral radius of the matrix $\left[F\left(E_{0}\right)\right]\left[V\left(E_{0}\right)\right]^{-1}$ denoted and defined by [20]:

Then from $\lambda_{1}, \lambda_{2}, \lambda_{3}$ the dominant eigenvalue is $\lambda_{1}=\frac{\beta \eta(p q c+(1-p) d)}{b c d}$.

Therefore the basic reproduction number is given by

$$
R_{0}=\frac{\beta \eta(p q(\mu+\gamma+\zeta)+(\alpha+\mu+\zeta)(1-p))}{(\eta+\mu+\varphi)(\mu+\gamma+\zeta)(\alpha+\mu+\zeta)(\mu+\theta)} .
$$

\section{). Endemic Equilibrium Points}

The endemic equilibrium point denoted by $E_{1}=\left\{S^{*}, E^{*}, A^{*}, I^{*}, Q^{*}\right\}$ is a steady state solution where the disease persists in the population. The endemic equilibrium point of a model is a solution of the system of equation. Then solving the system of differential equation 2.1 by substitution and after some algebraic simplificaton we obtain: $E_{1}=\left\{S^{*}, E^{*}, A^{*}, I^{*}, Q^{*}\right\}$ as: 


$$
\begin{aligned}
& S^{*}=\frac{N^{*}}{R_{0}}, \\
& E^{*}=\frac{\pi c d e}{b c d e-[p c \alpha \eta \theta+(1-p) \gamma \eta \theta d+\varphi \theta d c]}\left(1-\frac{1}{R_{0}}\right), \\
& A^{*}=\frac{(1-p) \pi \eta d e}{b c d e-[p c \alpha \eta \theta+(1-p) \gamma \eta \theta d+\varphi \theta d c]}\left(1-\frac{1}{R_{0}}\right), \\
& I^{*}=\frac{\pi \eta p c e}{b c d e-[p c \alpha \eta \theta+(1-p) \gamma \eta \theta d+\varphi \theta d c]}\left(1-\frac{1}{R_{0}}\right), \\
& Q^{*}=\frac{\pi}{\theta}\left[\frac{b c d e}{b c d e-[p c \alpha \eta \theta+(1-p) \gamma \eta \theta d+\varphi \theta d c]}+1\right](1- \\
& \left.\frac{1}{R_{0}}\right), \\
& \text { where, } b=\eta+\mu+\varphi, c=\mu+\gamma+\zeta, \\
& d=\alpha+\mu+\zeta, \quad e=\theta+\mu .
\end{aligned}
$$

From the above solution of the model we conclude that a positive unique endemic quilibrium point exists whenever $R_{0}>1$.

\section{* Local Stability of Disease Free Equilibrium Points}

Theorem 2. The disease free equilibrium point, $E_{0}$ of the system (2.1) is locally asymptotically stable if $R_{0}<1$ and unstable otherwise.

Proof: Consider the right hand side expressions of the equations (2.1) as functions so as to find the Jacobian matrix at the DFE as:

$$
J\left(E_{0}\right)=\left[\begin{array}{ccccc}
-\mu & 0 & -\beta q & -\beta & \theta \\
0 & -b & \beta q & \beta & 0 \\
0 & (1-p) \eta & -c & 0 & 0 \\
0 & p \eta & 0 & -d & 0 \\
0 & \varphi & \gamma & \alpha & -e
\end{array}\right]
$$

Where $b=\mu+\eta+\varphi, c=\mu+\gamma+\zeta$,

$d=\mu+\alpha+\zeta, e=\theta+\mu$

The characteristic equation of the Jacobian matrix at the disease free equilibrium point is given by $(\mu+\lambda)(e+\lambda)\left(\lambda^{3}+a_{1} \lambda^{2}+a_{2} \lambda+a_{3}\right)=0$, where

$a_{1}=b+c+d=3 \mu+2 \zeta+\eta+\varphi+\alpha+\gamma$, $a_{2}=b c+b d+c d-\beta \eta[p(1-q)+q]$

$=3 \mu^{2}+\mu(2 \eta+2 \varphi+2 \gamma+2 \alpha+3 \zeta)+\zeta^{2} 2+$ $\zeta(2 \gamma+2 \eta+2 \varphi+\alpha)+\gamma(\eta+\varphi+\alpha)+\alpha(\eta+$ $\varphi)-\beta \eta[p(1-q)+q]$, $a_{3}=b c d\left(1-R_{0}\right)=(\mu+\eta+\varphi)(\mu+\gamma+\zeta)(\mu+$

$\alpha+\zeta)\left(1-R_{0}\right)$,

Then, $(\mu+\lambda)(e+\lambda)=0$ or

$\lambda^{3}+a_{1} \lambda^{2}+a_{2} \lambda+a_{3}=0$

From this the first two eigenvalues

$\lambda_{1}=-\mu, \lambda_{2}=-e$, are real, distinct and negative.

To determine the sign of the three left eigenvalues we use the Routh-Hurwitz criteria for the cubic equation; $\lambda^{3}+a_{1} \lambda^{2}+a_{2} \lambda+a_{3}=0$. According to the Routh-Hurwitz criteria the three roots of a polynomial of order three of type $p(\lambda)=$ $\lambda^{3}+a_{1} \lambda^{2}+a_{2} \lambda+a_{3}$, are real distinct and negative if the coefficients satisfy the conditions $a_{1}>0, a_{2}>0, a_{3}>0$ and $a_{1} a_{2}>a_{3}$

It is straight forward to verify that this conditions are satisfied and hence the last three eigenvalues are real typical and negative.i.e

$a_{1}>0 \Rightarrow b+c+d>0$

$a_{2}>0 \Rightarrow b c+b d+c d>\beta \eta[p(1-q)+q]$

$a_{3}>0 \Rightarrow \operatorname{bcd}\left(1-R_{0}\right)>0 \Rightarrow R_{0}<1$ and

$a_{1} a_{2}>a_{3} \Rightarrow(b+c+d)(b c+b d+c d+\beta q \eta(p-$ 1) $-\beta p \eta)>b c d\left(1-R_{0}\right)$.

$=b^{2}(c+d)+c^{2}(b+d)+d^{2}(b+c)+3 b c d-$ $\beta \eta(b+c+d)(p+q(1-p))>b c d\left(1-R_{0}\right)$

Therefore the disease free equilibrium point of the system of ordinary differential equation (2.1) is locally asymptotically stable if $R_{0}<1$.

+. Global Stability of the Disease Free Equilibrium Point

Theorem 3. The disease free equilibrium point $E_{0}$ of the model equation 2.1 is globally asymptotically stable if $R_{0}<1$.

Proof: To establish the global stability of the disease-free equilibrium point, we construct a Lyapunov function. Let $\Omega \subseteq \mathbb{R}_{+}^{5}$ be an open neighborhood of the disease free equilibrium point $E_{0}$ of the function; 
$L: \Omega \longrightarrow \mathbb{R}\left(L\left(E_{0}\right)=0\right)$.

Then the function $L$, defined by: $L(S, E, A, I, Q)=\frac{B_{1}}{2}(E(t))^{2}+\frac{B_{2}}{2}(A(t))^{2}+$ $\frac{B_{3}}{2}(I(t))^{2}$

where $B_{i}$, for $i=1,2,3$ are some positive constants to be chosen later.

Then $L(S, E, A, I, Q)$ should satisfies the following properties to be a lyapunov function:

i) $L$ is continuously differentiable.

ii) $L>0, \forall x \in \Omega \backslash E_{0}$ and $L\left(E_{0}\right)=0$, as $(E(t))^{2} \geq 0,(A(t))^{2} \geq 0,(I(t))^{2} \geq 0$. iii) $\frac{d L}{d t} \leq 0$ in $\Omega$, then $E_{0}$ is stable.

The first two condition holds, as L it is continuously differentiable and $L>0, \forall x \in \Omega \backslash E_{0}$ and $L\left(E_{0}\right)=0$. Now let we check the third condition $\frac{d L}{d t} \leq 0$ in $\Omega$.

$\frac{d L}{d t}=B_{1} \frac{d E}{d t}+B_{2} \frac{d A}{d t}+B_{3} \frac{d I}{d t}$
$=B_{1}(\lambda S-(\mu+\eta+\varphi) E)+B_{2}((1-p) \eta E-$
$(\mu+\gamma+\zeta) A)+B_{3}(p \eta E-(\mu+\alpha+\zeta) I)$
$=B_{1}(\lambda S-\underbrace{(\mu+\eta+\varphi)}_{b} E)+B_{2}((1-p) \eta E-$
$\underbrace{(\mu+\gamma+\zeta)}_{c} A)+B_{3}(p \eta E-\underbrace{(\mu+\alpha+\zeta)}_{d} I)$
$=B_{1}(\lambda S-b E)+B_{2}((1-p) \eta E-c A)+$

$B_{3}(p \eta E-d I)$

$=B_{1}\left(\frac{\beta(I+q A)}{N} S-b E\right)+B_{2}((1-p) \eta E-c A)+$

$B_{3}(p \eta E-d I), S \leq N$ at $E_{0}$.

$=B_{1}(\beta(I+q A)-b E)+B_{2}((1-p) \eta E-$

$c A)+B_{3}(p \eta E-d I)$

$=B_{1} \beta I+B_{1} \beta q A-B_{1} b E+B_{2}(1-p) \eta E-$

$B_{2} c A+B_{3} p \eta E-B_{3} d I$

$=\left(B_{3} p \eta+B_{2}(1-p) \eta-B_{1} b\right) E+\left(B_{1} \beta-\right.$

$\left.B_{3} d\right) I+\left(B_{1} \beta q-B_{2} c\right) A$

$=B_{1} b\left(\frac{\left(B_{3} p \eta+B_{2}(1-p) \eta\right.}{B_{1} b}-1\right) E+\left(B_{1} \beta-B_{3} d\right) I+$

$\left(B_{1} \beta q-B_{2} c\right) A$.

Now choosing $B_{3}=\beta c, B_{2}=\beta d q, B_{1}=c d$.

Then, $\frac{d L}{d t}=b c d\left(\frac{\beta c p \eta+\beta q d(1-P) \eta}{b c d}-1\right) E+$

$(c d \beta-\beta c d) I+(c d \beta q-\beta d q c) A$

$=b c d\left(\frac{\beta c p \eta+\beta q d(1-p) \eta}{b c d}-1\right) E+0$

$=b c d \underbrace{\left(\frac{(p c+q d(1-p)) \beta \eta}{b c d}\right.}_{R_{0}}-1) E$

$=\operatorname{bcd}\left(R_{0}-1\right) E$

Therefore $\frac{d L}{d t} \leq b c d\left(R_{0}-1\right) E<0$ if $R_{0}-1<0$.

Thus, this is true when $R_{0}<1$ which implies that $\frac{d L}{d t}$ is negative. Therefore the largest compact invariant set in $\Omega$ is singleton set $E_{0}$. Hence LaSalle's invariant principle [15] implies that $E_{0}$ is globally asymptotically stable.

\section{,. Local Stability of Endemic Equilibrium Point}

Theorem 4. The endemic equilibrium point $E_{1}=\left(S^{*}, E^{*}, A^{*}, I^{*}, Q^{*}\right)$ of the system 2.1 is locally asymptotically stable if $R_{0}>1$.

Proof: The characteristic equation of the Jacobian matrix (3.7) at the endemic equlibrium point is given by

$P(\lambda)=\lambda^{5}+B_{1} \lambda^{4}+B_{2} \lambda^{3}+B_{3} \lambda^{2}+B_{4} \lambda+B_{5}=0$

where the notation of the coefficients are:

$B_{1}=b+c+d+e+k+\mu$

$B_{2}=b c+b d+c d+e k+\mu e+(e+k+\mu)(b+$

$c+d)-\frac{\beta \eta S^{*}}{N^{*}}[p(1-q)+q]$

$B_{3}=(b+c+d)+(e+k+\mu)(b c+b d+c d+(b+$

$c+d)(e k+\mu e)-\frac{S^{*}}{N^{*}}\left[R_{0} b c d+\beta \eta(p(1-q)+q)\right]$

$B_{4}=(e k+\mu e)\left(b c+b d+c d+\frac{\beta \eta S^{*}}{N^{*}}(p q-p-\right.$

$q))-\frac{R_{0} b c d S^{*}}{N^{*}}(e+k+\mu)$

$B_{5}=\frac{\beta \eta S^{*} e}{N^{*}}(k+\mu)(p q d-p c-q d)$

where $k=\left\{\frac{\beta\left(I^{*}+q A^{*}\right)}{N^{*}}=\right.$

$\left.\frac{b c d e \pi R_{0}}{N^{*}[b c d e-[p c \alpha \eta \theta+(1-p) \gamma \eta \theta d+\varphi \theta d c]]}\left(1-\frac{1}{R_{0}}\right)\right\}$

Then the eigenvalues of the characteristic 
equation (3.8) will be negative if it fulfill the Routh-Hurwitz criteria [15] that are $B_{i}>0$ for $i=1,2,3,4,5$ and the determinant of $p(\lambda)>0$ that is

From the Routh - Hurwitz criteria [15] the determinant of the Hurwitz matrix becomes non negative if the following conditions holds true and it follows that all eigenvalues of the characteristic equation (3.8) has negative real part if and only if $B_{i}>0,(i=1,2,3,4,5)$ which implies that

(i) $B_{1}>0$ Since all the parameters in the models are positives.

(ii) $B_{2}>0 \Leftrightarrow b c+b d+c d+e k+\mu e+(e+$ $k+\mu)(b+c+d)+\frac{\beta \eta q p}{R_{0}}>\frac{\beta \eta}{R_{0}}(p+q)$

(iii) $B_{3}>0 \Leftrightarrow(b+c+d)+(e+k+\mu)(b c+$ $b d+c d+(b+c+d)(e k+\mu e)+\frac{\beta \eta}{R_{0}}(p q d+p q)>$ $\frac{\beta \eta}{R_{0}}(p c+q d+p+q)$

(iv) $B_{4}>0 \Leftrightarrow e(k+\mu)\left(b c+b d+c d+\frac{\beta \eta q p}{R_{0}}\right)+$ $(e+k+\mu)\left(\frac{\beta \eta q p d}{R_{0}}\right)>\frac{\beta \eta}{R_{0}} e(k+\mu)(p+q)+\frac{\beta \eta}{R_{0}}(e+$ $k+\mu)(p c+q d)$

(v) $B_{5}>0 \Leftrightarrow e\left(\frac{\beta \eta q p d}{R_{0}}\right)(k+\mu)>e \frac{\beta \eta q}{R_{0}}(k+$ $\mu)(p c+q d))$.

and the determinant,

$D_{1}=B_{1}>0$

$D_{2}=B_{1} B_{2}>0$

$D_{3}=B_{1} B_{2}-B_{3}>0$

$D_{4}=B_{1} B_{2} B_{3}-B_{3}^{2}-B_{1}^{2} B_{4}>0$

$D_{5}=B_{1} B_{2} B_{3} B_{4}+2 B_{1} B_{4} B_{5}+B_{2} B_{3} B_{5}-$ $B_{1} B_{2}^{2} B_{5}-B_{1}^{2} B_{4}^{2}-B_{3}^{2} B_{4}-B_{5}^{2}>0$.

Therefore the system (2.1) shows local asymptotic stability at $E_{1}$ when $R_{0}>1$ and conditions for $D_{1}, D_{2}, D_{3}, D_{4}, D_{5}$ are satisfied.

-. Global Stability of the Endemic Equilibrium Point

Theorem 5. The endemic equilibrium point of the model equation(2.1) is globally asymptotically stable if $R_{0}>1$.
Proof: To show the result, we define the following

Lyapunov function as:

$$
\begin{aligned}
L\left(S^{*}, E^{*}, A^{*}, I^{*}, Q^{*}\right)= & {\left[S-S^{*}-S^{*} \ln \left(\frac{S}{S^{*}}\right)\right] } \\
& +\left[E-E^{*}-E^{*} \ln \left(\frac{E}{E^{*}}\right)\right] \\
& +\left[A-A^{*}-A^{*} \ln \left(\frac{A}{A^{*}}\right)\right] \\
& +\left[I-I^{*}-I^{*} \ln \left(\frac{I}{I^{*}}\right)\right] \\
& +\left[Q-Q^{*}-Q^{*} \ln \left(\frac{Q}{Q^{*}}\right)\right]
\end{aligned}
$$

Then by taking the time derivative of $L\left(S^{*}, E^{*}, A^{*}, I^{*}, Q^{*}\right)$, we obtain:

$\frac{d L}{d t}=\left(S^{\prime}-\frac{S^{*}}{S} S^{\prime}\right)+\left(E^{\prime}-\frac{E^{*}}{E} E^{\prime}\right)+\left(A^{\prime}-\right.$ $\left.\frac{A^{*}}{A} A^{\prime}\right)+\left(I^{\prime}-\frac{I^{*}}{I} I^{\prime}\right)+\left(Q^{\prime}-\frac{Q^{*}}{Q} Q^{\prime}\right)$

$=\left(1-\frac{S^{*}}{S}\right) \frac{d S}{d t}+\left(1-\frac{E^{*}}{E}\right) \frac{d E}{d t}+\left(1-\frac{A^{*}}{A}\right) \frac{d A}{d t}+$ $\left(1-\frac{I^{*}}{I}\right) \frac{d I}{d t}+\left(1-\frac{Q^{*}}{Q}\right) \frac{d Q}{d t}$

$=\left[1-\frac{S^{*}}{S}\right][\pi+\theta Q-(\lambda+\mu) S]+\left[1-\frac{E^{*}}{E}\right][\lambda S-$ $(\mu+\eta+\varphi) E]+\left[1-\frac{A^{*}}{A}\right][(1-p) \eta E-(\mu+$ $\gamma) A]+\left[1-\frac{I^{*}}{I}\right][p \eta E-(\mu+\alpha+) I]+[1-$ $\left.\frac{Q^{*}}{Q}\right][\alpha I+\gamma A+\varphi E-(\theta+\mu) Q]$

$=\left(\pi+\theta Q+\lambda S^{*}+\mu S^{*}-\lambda S-\mu S-\pi \frac{S^{*}}{S}-\right.$ $\left.\theta Q \frac{S^{*}}{S}\right)+\left(\lambda S+\mu E^{*}+\eta E^{*}+\varphi E^{*}-\mu E-\eta E-\right.$ $\left.\varphi E-\lambda S \frac{E^{*}}{E}\right)+\left(\eta E+\mu A^{*}+\gamma A^{*}+\frac{A^{*}}{A} p \eta E-\right.$ $\left.p \eta E-\mu A-\gamma A-\frac{A^{*}}{A} \eta E\right)+\left(p \eta E \mu I^{*}+\alpha I^{*}-\right.$ $\left.\mu I-\alpha I-\frac{I^{*}}{I} p \eta E\right)+\left(\alpha I+\gamma I+\varphi E+\theta Q^{*}+\right.$ $\left.\mu Q^{*}-\theta Q-\mu Q-\alpha I \frac{Q^{*}}{Q}-\gamma A \frac{Q^{*}}{Q}-\varphi E \frac{Q^{*}}{Q}\right)$

Now after some simplifications

$\frac{d L}{d t}=[\pi+(\lambda+\mu) S^{*}+\underbrace{(\mu+\eta+\varphi)}_{b} E^{*}+$

$\frac{A^{*}}{A} p \eta E+\underbrace{(\mu+\gamma)}_{c} A^{*}+\underbrace{(\mu+\alpha)}_{d} I^{*}+$ $\underbrace{(\theta+\mu)}_{e} Q^{*}]-[\underbrace{(S+E+A+I+Q)}_{N} \mu+$ $\theta Q \frac{S^{*}}{S}+\lambda S \frac{E^{*}}{E}+\frac{A^{*}}{A} \eta E+\frac{I^{*}}{I} p \eta E+\alpha I \frac{Q^{*}}{Q}+$ $\left.\gamma A \frac{Q^{*}}{Q}+\varphi E \frac{Q^{*}}{Q}\right]$

$=\left[\pi+(\lambda+\mu) S^{*}+b E^{*}+p \eta E \frac{A^{*}}{A}+c A^{*}+\right.$ $\left.d I^{*}+e Q^{*}\right]-\left[N \mu+\theta Q \frac{S^{*}}{S}+\lambda S \frac{E^{*}}{E}+\eta E \frac{A^{*}}{A}+\right.$ $\left.p \eta E \frac{I^{*}}{I}+(\alpha I+\gamma A+\varphi E) \frac{Q^{*}}{Q}\right], N \leq \frac{\pi}{\mu}$ $=\left[\pi+(\lambda+\mu) S^{*}+b E^{*}+p \eta E \frac{A^{*}}{A}+c A^{*}+\right.$ 
$\left.d I^{*}+e Q^{*}\right]-\left[\pi+\theta Q \frac{S^{*}}{S}+\lambda S \frac{E^{*}}{E}+\eta E \frac{A^{*}}{A}+\right.$ $\left.p \eta E \frac{I^{*}}{I}+\alpha I \frac{Q^{*}}{Q}+\gamma A \frac{Q^{*}}{Q}+\varphi E \frac{Q^{*}}{Q}\right]$

$=\left[(\lambda+\mu) S^{*}+b E^{*}+p \eta E \frac{A^{*}}{A}+c A^{*}+d I^{*}+\right.$ $\left.e Q^{*}\right]-\left[\theta Q \frac{S^{*}}{S}+\lambda S \frac{E^{*}}{E}+\eta E \frac{A^{*}}{A}+p \eta E \frac{I^{*}}{I}+\right.$ $\left.\alpha I \frac{Q^{*}}{Q}+\gamma A \frac{Q^{*}}{Q}+\varphi E \frac{Q^{*}}{Q}\right]$

$\frac{d L}{d t}=M-K$

where $M=(\lambda+\mu) S^{*}+b E^{*}+p \eta E \frac{A^{*}}{A}+c A^{*}+$ $d I^{*}+e R^{*}$

$K=\theta Q \frac{S^{*}}{S}+\lambda S \frac{E^{*}}{E}+\eta E \frac{A^{*}}{A}+p \eta E \frac{I^{*}}{I}+\alpha I \frac{Q^{*}}{Q}+$ $\gamma A \frac{Q^{*}}{Q}+\varphi E \frac{Q^{*}}{Q}$

$N=S+E+A+I+Q$ and

$N^{*}=S^{*}+E^{*}+A^{*}+I^{*}+Q^{*}$

Thus if $K<M$, then $\frac{d L}{d t}<0$. Nothing that $\frac{d L}{d t}=0$ if and only if

$S=S^{*}, E=E^{*}, A=A^{*}, I=I^{*}, Q=Q^{*}$.

Therefore the largest compact invariant set in $\left\{\left(S^{*}, E^{*}, A^{*}, I^{*}, Q^{*}\right) \in \Omega ; \frac{d L}{d t}=0\right\}$ is a singleton $E_{1}$ is the endemic equilibrium point of the system (2.1). By LaSalle's invariant principle [15], it implies that $E_{1}$ is globally asymptotically stable in $\Omega$ if $M<k$ and $R_{0}>1$.

\section{SENSITIVITY ANALYSIS}

Sensitivity analysis allows us to assess the impact that changes in a certain parameter will have on the model and it can help some one to determine which parameters are the key drivers of a model's results. To investigate which parameters have high impact on the $R_{0}$, we apply the approach presented in [18]. The main goal of this section is to perform sensitivity analysis of corona virus transmission dynamic model to the parameters describing in it. We perform sensitivity analysis by calculating the sensitivity indices of the basic reproduction number $R_{0}$ in order to determine whether COVID-19 can be spread in the population or not. These indices tell us how crucial each parameter is on the transmission of the COVID-19. Following [17, 21], we used the normalized forward sensitivity index also called elasticity as it is the backbone of nearly all other sensitivity analysis techniques [21] and are computationally efficient[22]. For instance, the normalized forward sensitivity index of the basic reproduction number, $R_{0}$ with respect to a parameter value, $P$ is given by:

$$
\Upsilon_{p}^{R_{0}}=\frac{\partial R_{0}}{\partial P} \times \frac{P}{R_{0}} .
$$

The explicit expression of $R_{0}$ is given by

$$
R_{0}=\frac{\beta \eta(p q(\mu+\gamma+\zeta)+(\alpha+\mu+\zeta)(1-p))}{(\eta+\mu+\varphi)(\mu+\gamma+\zeta)(\alpha+\mu+\zeta)(\mu+\theta)} .
$$

Since $R_{0}$ depends only on ten parameters, we derive an analytical expression for its sensitivity to each parameter using the normalized forward sensitivity index as in [18] by taking the values of the paramters from table 2 and computed as follows:

$$
\Upsilon_{\beta}^{\left(R_{0}\right)}=\frac{\partial R_{0}}{\partial \beta} \times \frac{\beta}{R_{0}}=1,
$$

$$
\Upsilon_{\eta}^{\left(R_{0}\right)}=\frac{\partial R_{0}}{\partial \eta} \times \frac{\eta}{R_{0}}=\frac{\mu+\varphi}{\eta+\mu+\varphi}
$$

$$
\begin{aligned}
& \Upsilon_{p}^{\left(R_{0}\right)}= \\
& \frac{[q(\mu+\gamma+\zeta)-(\alpha+\mu+\zeta)] p}{p q(\mu+\gamma+\zeta)+(\alpha+\mu+\zeta)(1-p)} \\
& \frac{\partial R_{0}}{\partial p}
\end{aligned}
$$

$\frac{\Upsilon_{q}^{\left(R_{0}\right)}=}{\frac{\partial R_{0}}{\partial q}} \times \frac{q}{R_{0}}=$

$$
\begin{gathered}
\Upsilon_{\mu}^{\left(R_{0}\right)}=\frac{\partial R_{0}}{\partial \mu} \times \frac{\mu}{R_{0}}= \\
\frac{[p+q(1-p)][(\eta+\mu+\varphi)(\mu+\gamma+\zeta)(\mu+\alpha+\zeta)]}{(\eta+\mu+\varphi)(\mu+\gamma+\varphi)(\mu+\alpha+\zeta)(\mu+\theta)}- \\
\frac{[p(\mu+\gamma+\zeta)+q(\alpha+\mu+\zeta)(1-p)]}{(\eta+\mu+\varphi)(\mu+\gamma+\varphi)(\mu+\alpha+\zeta)(\mu+\theta)} \times \\
\left.\frac{[(\mu+\gamma+\zeta)(\mu+\alpha+\zeta)+(\eta+\mu+\varphi)(\mu+\alpha+\zeta)+(\eta+\mu+\varphi)(\mu+\gamma+\zeta)]}{(\eta+\mu+\varphi)(\mu+\gamma+\varphi)(\mu+\alpha+\zeta)(\mu+\theta)}\right] \times \\
\frac{\mu}{p(\mu+\gamma+\zeta)+q(\alpha+\mu+\zeta)(1-p)}, \\
\Upsilon_{\varphi}^{\left(R_{0}\right)}=\frac{\partial R_{0}}{\partial \varphi} \times \frac{\varphi}{R_{0}}=\frac{-\varphi}{\mu+\eta+\varphi},
\end{gathered}
$$




$$
\begin{gathered}
\Upsilon_{\zeta}^{\left(R_{0}\right)}=\underset{\frac{\left[p R_{0}\right.}{\partial \zeta}}{\frac{[p q+(1-p)][(\mu+\gamma+\zeta)(\mu+\alpha+\zeta)]}{(\alpha+\mu+\zeta)(\mu+\gamma+\zeta)}-} \times \frac{\zeta}{R_{0}} \\
\left.\frac{[p q(\mu+\gamma+\zeta)+(\alpha+\mu+\zeta)(1-p)][2 \mu+2 \zeta+\alpha+\gamma]}{(\alpha+\mu+\zeta)(\mu+\gamma+\zeta)}\right) \\
\times \frac{\zeta}{p q(\mu+\gamma+\zeta)+(\alpha+\mu+\zeta)(1-p)}, \\
=\frac{\partial R_{0}}{\partial \gamma} \times \frac{\gamma}{R_{0}} \\
\Upsilon_{\gamma}^{\left(R_{0}\right) \quad}= \\
\frac{-(\alpha+\mu+\zeta)(1-p) \gamma}{(\mu+\gamma+\zeta)[p q(\mu+\gamma+\zeta)+(\alpha+\mu)(1-p)]}, \\
\Upsilon_{\alpha}^{\left(R_{0}\right) \quad \frac{\partial R_{0}}{\partial \alpha}} \times \frac{\alpha}{R_{0}} \\
\frac{-p q(\mu+\gamma+\zeta)}{(\mu+\alpha+\zeta)[p q(\mu+\gamma+\zeta)+(\alpha+\mu+\zeta)(1-p)]} \cdot \\
\Upsilon_{\theta}^{\left(R_{0}\right)}=\frac{\partial R_{0}}{\partial \theta} \times \frac{\eta}{R_{0}}=\frac{-\theta}{\mu+\theta},
\end{gathered}
$$

\section{EXTENSION OF COVID-19 MODEL INTO AN OPTIMAL CONTROL}

In this section, our main focus is to setup an optimal control problem relative to the epidemic model (2.1). We introduce to SEAIQ epidemic model 2.1 a control function $u_{1}(t)$ that represent the effort of preventation(vaccination, using mask, hand washing, keeping social distance etc) to prevent susceptible individuals from exposing to COVID-19 disease. Thus, the transmission dynamic of COVID-19 model with optimal control is governed by nonlinear system of differential equations as follows:

$$
\left\{\begin{array}{l}
\frac{d S}{d t}=\pi+\theta Q-\left(1-u_{1}\right) \lambda S-\mu S, \\
\frac{d E}{d t}=\left(1-u_{1}\right) \lambda S-\eta E-\varphi E-\mu E \\
\frac{d A}{d t}=(1-p) \eta E-\gamma A-(\mu+\zeta) A \\
\frac{d I}{d t}=p \eta E-\alpha I-(\mu+\zeta) I \\
\frac{d R}{d t}=\alpha I+\gamma A+\varphi E-(\theta+\mu) Q
\end{array}\right.
$$

$S(0)=S_{0}>0, E(0)=E_{0} \geq 0, A(0)=A_{0} \geq$ $0, I(0)=I_{0} \geq 0, Q(0)=Q_{0} \geq 0$.
Table 1: Sensitivity Indices of the Basic Model Parameters in $R_{0}$

\begin{tabular}{|c|c|}
\hline \hline Parameter Symbol & Sensitivity indices \\
\hline$\beta$ & +1 \\
\hline$\eta$ & 0.9943 \\
\hline$q$ & 0.1273 \\
\hline$p$ & 0.0646 \\
\hline$\theta$ & -0.0099 \\
\hline$\zeta$ & -0.0151 \\
\hline$\alpha$ & -0.0549 \\
\hline$\mu$ & -0.3488 \\
\hline$\gamma$ & -0.6288 \\
\hline$\varphi$ & -0.9470 \\
\hline
\end{tabular}

From table[1] above those parameters that have positive indices, $\beta, \eta, q$ and $p$ show that they have great impact on expanding the disease in the population if their values are increasing. Because the basic number of copies increases as the value increases.

Furthermore, those parameters in which their sensitivity indices are negative, $\theta, \zeta, \alpha, \mu, \gamma$ and $\varphi$ have an influence of minimizing the endemic nature of the disease in the community due to the reason that the basic reproduction number decreases as their values decrease.

Where $\lambda=\frac{\beta(I+q A)}{N}$ is the force of infection in the model to control.

The optimal control problem is to minimize the objective (cost functional) $(J)$ considering the costs of preventation(vaccination, using mask, hand washing, keeping social distance etc) of susceptible individuals over a fixed period of time $T$. Mathematically, the optimal control problem consists of minimizing the objective functional $J$, on a fixed time interval $T$ takes the form;

$J\left(u_{1}(t)\right)=\int_{0}^{T}\left[M_{1} E+M_{2} A+M_{3} I+M_{4} Q+\frac{1}{2} k_{1} u_{1}^{2}(t)\right] d t \longrightarrow \min$

subject to 


$$
g(x, u, t)=\left\{\begin{array}{l}
\frac{d S}{d t}=\pi+\theta Q-\left(1-u_{1}\right) \frac{\beta}{N}(I+q A) S-\mu S, \\
\frac{d E}{d t}=\left(1-u_{1}\right) \frac{\beta}{N}(I+q A) S-\eta E-\varphi E-\mu E, \\
\frac{d A}{d t}=(1-p) \eta E-\gamma A-(\mu+\zeta) A, \\
\frac{d I}{d t}=p \eta E-\alpha I-(\mu+\zeta) I, \\
\frac{d R}{d t}=\alpha I+\gamma A+\varphi E-(\theta+\mu) Q,
\end{array}\right.
$$

with initial condition, $S(0)=S_{0}>0$,

$$
\begin{aligned}
& E(0)=E_{0} \geq 0, A(0)=A_{0} \geq 0, \\
& I(0)=I_{0} \geq 0, Q(0)=Q_{0} \geq 0 \text { and } \\
& \quad \Omega_{\epsilon}=\left\{u_{1}: 0 \leq u_{1} \leq 1, t \in[0, T]\right\},
\end{aligned}
$$

where $M_{1}, M_{2}, M_{3}, M_{4}, \frac{k_{1}}{2}$ are positive weights that balance the size of the integrand terms to reduce the dominance of any of the term in the integral. Such that the constant weight $k_{1}$ measures the cost or effort required for the implementation of prevention while $M_{1}, M_{2}, M_{3}$ and $M_{4}$ measures the relative importance of reducing the associated classes on the spread of the disease. The parameter $T$ is the duration of time, in a days, of prevantion.

We assumed that $0 \leq u_{1}<1$, since avoiding the contact between the entire susceptible and infectious individuals are impossible in reality. In practice, preventing the entire society is impossible due to many factors such as financial and human resource constraint. If $u_{1}=0$ then no control measure is taken and the model equation (5.11) is equivalent to (2.1). On the other hand, if $u_{1}=1$, it implies our control is $100 \%$ success. But in the reality this case is not possible and our aim is to minimize the number of exposed, infected, asymptomatic , quarantine and cost. Thus, we seek to find an optimal controls $\left(u_{1}^{*}\right)$ such that

$$
J\left(u_{1}^{*}\right)=\min \left\{J\left(u_{1} \in \Omega_{\epsilon}\right\}\right.
$$

where $\Omega_{\epsilon}$ is the set of admissible control as defined above.
The solution of the optimal control problem is the vector function $x^{*}()=$. $\left(S^{*}(),. E^{*}(),. A^{*}(),. I^{*}(),. Q^{*}().\right) \in \mathbb{R}_{+}^{5}$, associated with an admissible control $u^{*}=u_{1}^{*} \in \Omega_{\epsilon}$ on the time interval $[0, T]$ that minimize the cost functional 5.12) where $u_{1}^{*}$ are Lebesgue measurable function on $[0,1]$ that is the control $u_{1}^{*}$ are piecewise continuous and integrable.

\section{A. Existence of Optimal Control Solution}

Theorem 6. There exist an optimal control $u^{*}=$ $u_{1}^{*}$ and a corresponding solution vector $x^{*}$ to the state initial value problem (5.11) that minimizes the cost functional $J(u)$ of (5.12) over the set of admissible control $\Omega_{\epsilon}$.

Proof : The non trivial requirement on the set of admissible controls and the set of end conditions are followed by [23].

$i$. The set of all solution to system (5.11) with corresponding control functions in $\Omega_{\epsilon}$ is non-empty.

ii. The control unit is convex and closed

iii. The right hand side of the state system is bounded by a linearized function in the state and control variables.

$i v$. The integral of the objective function is convex.

v. There exist a constants $c_{1}, c_{2}>0$ and $\beta>1$ such that the integrand of the objective functional 
satisfies, $M_{1} E+M_{2} A+M_{3} I+M_{4} Q+\frac{k_{1}}{2} u_{1}^{2} \geq$ $c_{1}\left(\left|u_{1}\right|^{2}\right)^{\frac{\beta}{2}}-c_{2}$.

In order to established condition (i), we consider the Picard-Lindelof existence theorem [23]. If $g(x, u, t)$ is bounded, continuous, and lipschitz in the state variables, then there exists a unique solution corresponding to every admissible control $\Omega_{\epsilon}$. Hence, for any $u \in \Omega_{\epsilon}$ and the state variables, we have

$$
0<N \leq \frac{\pi}{\mu}
$$

and non empty by model assumption. Furthermore, with the bounded established (5.15) it implies that the state system is continuous and bounded. It is possible to show the boundedness of the partial derivative with respect to the state variable i.e $\frac{\partial g}{\partial x}$ exist and finite, which established the system is Lipschitz with respect to the state variables. This established the proof of condition (i).

For condition consider

$\Omega_{\epsilon}=\{u \in R:\|u\| \leq 1-\varepsilon$.

Assume that $u_{1} \in \Omega_{\epsilon}$ and $\lambda \in[0,1]$, such that $\left\|u_{1}\right\| \leq 1-\varepsilon$.

Hence, $0 \leq \lambda\left\|u_{1}\right\| \leq 1-\varepsilon$, for $u_{1} \in \Omega_{\epsilon}$ and $\lambda \in[0,1]$.

Thus the control set is convex and closed by definition.

To prove condition (iii), we consider the system (5.11). Then

$$
\begin{gathered}
\frac{d N}{d t}=\frac{d S}{d t}+\frac{d E}{d t}+\frac{d A}{d t}+\frac{d I}{d t}+\frac{d Q}{d t}, \\
=\pi-\mu N-\zeta(A+I) \leq \pi-\mu N \\
\frac{d N}{d t}+\mu N \leq \pi \\
\lim _{t \rightarrow \infty} \sup N(t) \leq \frac{\pi}{\mu} .
\end{gathered}
$$

Therefore, all solutions of the model (5.11) are bounded. We can write system (5.11) in matrix form as

$$
\begin{gathered}
F(t, x, u)=G(t, x)+H(t, x) U, \\
\text { where }
\end{gathered}
$$

$$
\begin{gathered}
F(t, x, u)=\left[\begin{array}{c}
\pi+\theta Q-\left(1-u_{1}\right) \lambda S-\mu S \\
\left(1-u_{1}\right) \lambda S-\eta E-\varphi E-\mu E \\
(1-p) \eta E-\gamma A-(\mu+\zeta) A \\
p \eta E-\alpha I-(\mu+\zeta) I \\
\alpha I+\gamma A+\varphi E-(\theta+\mu) Q
\end{array}\right], \\
G(t, x)=\left[\begin{array}{c}
\pi+\theta Q-(\lambda+\mu) S \\
\lambda S-(\mu+\eta+\varphi) E \\
(1-p) \eta E-(\mu+\gamma+\zeta) A \\
p \eta E-(\mu+\alpha+\zeta) I \\
\alpha I+\gamma A+\varphi E-(\theta+\mu) Q
\end{array}\right] \\
H(t, x)=\left[\begin{array}{ccc}
\lambda S & 0 & 0 \\
-\lambda S & (\eta-1) E & 0 \\
0 & -(1-p) \eta E & -A \\
0 & -p \eta E & -I \\
0 & E & I+A
\end{array}\right] \text { and } \\
\text { and state variable. } \\
U=\left[\begin{array}{c}
u_{1} \\
0 \\
0
\end{array}\right], \text { It gives a linar function of control }
\end{gathered}
$$

Then the norm of $F(t, x, u)=G(t, x)+$ $H(t, x) U$ is

$$
\begin{gathered}
\|F(t, x, u)\|=\|G(t, x)+H(t, x) U\| \\
\|F(t, x, u)\| \leq\|G(t, x)\|+\|H(t, x) U\| \\
\|F\| \leq\|G\|\|X(t)\|+\|H\|\|U(t)\| \leq \\
\max (\|G\|,\|H\|)
\end{gathered}
$$

This shows that the right hand side is bounded by the sum of the state and control variable. This complete the proof of condition (iii) which is bounded in the state and control variables.

(iv) The integrand in the objective functional which is a cost functional,

$$
g(x, t, u)=M_{1} E+M_{2} A+M_{3} I+M_{4} Q+\frac{1}{2} k_{1}^{2}
$$
is an affine function.

Recall that any affine function is a convex and the sum of a convex function is a convex [24]. Therefore, $g(x, t, u)$ is convex on $\Omega_{\epsilon}$. 


\section{Proof of condition (v)}

Given $g(x, t, u)=M_{1} E+M_{2} A+M_{3} I+$ $M_{4} Q+\frac{1}{2} k \cdot u_{1}^{2}(t)$.

$g(x, t, u) \geq \frac{1}{2} k_{1} u_{1}^{2}(t)$, $\beta=2$.

Thus, $\quad M_{1} E+M_{2} A+M_{3} I+M_{4} Q+\frac{k_{1}}{2} u_{1}^{2} \geq$ $c_{1}\left(\left|u_{1}\right|^{2}\right)^{\frac{\beta}{2}}-c_{2}$.

Since all the above conditions are satisfied, we conclude that there exists an optimal controls $u_{1}^{*}$ such that $\left(x, u^{*}\right)$ that minimize the cost functional of (5.12) over the set of admissible control.

\section{B. The nature of the optimal control solution}

Theorem 7. The optimal control problem (5.14) with fixed final time $T$ admits a unique optimal solution $\left(S^{*}, E^{*}, A^{*}, I^{*}, Q^{*}\right)$ associated with an optimal control $u=u_{1}$ for all $t \in[0, T]$. Moreover, there exist adjoint function $\lambda_{i}$,

for $i=1,2,3,4,5$ such that

$$
\left\{\begin{aligned}
\frac{d \lambda_{1}}{d t}= & \left(1-u_{1}\right) \frac{\beta}{N}(I+q A)\left(\lambda_{1}-\lambda_{2}\right)+\mu \lambda_{1} \\
\frac{d \lambda_{2}}{d t}=- & M_{1}+(\eta+\varphi+\mu) \lambda_{2}-(1-p) \eta \lambda_{3}- \\
& p \eta \lambda_{4}-\varphi \lambda_{5} \\
\frac{d \lambda_{3}}{d t}=- & M_{2}+\left(1-u_{1}\right) \frac{\beta}{N} q S\left(\lambda_{1}-\lambda_{2}\right)+ \\
& (\gamma+\mu+\zeta) \lambda_{3}-\gamma \lambda_{5} \\
\frac{d \lambda_{4}}{d t}=- & M_{3}+\left(1-u_{1}\right) \frac{\beta}{N} S\left(\lambda_{1}-\lambda_{2}\right)+ \\
& (\alpha+\mu+\zeta) \lambda_{4}-\alpha \lambda_{5} \\
\frac{d \lambda_{5}}{d t}=- & M_{4}+\theta\left(\lambda_{5}-\lambda_{1}\right)+\mu \lambda_{5}
\end{aligned}\right.
$$

with transiversality conditions

$\lambda_{i}(T)=0, \quad i=1,2,3,4,5$.

Furthermore, the following properties holds:

$$
u_{1}^{*}=\min \left\{\max \left\{0, \frac{\beta\left(I^{*}+q A^{*}\right) S^{*}\left(\lambda_{2}-\lambda_{1}\right)}{N k_{1}}\right\}, 1\right\}
$$

$\geq c_{1}\left[u_{1}^{2}(t)\right]^{\frac{\beta}{2}}-c_{2}$, where $c_{1}=\frac{1}{2} k_{1}, c_{2}>0$ and

with the system is defined as follows:

$$
\begin{aligned}
& H\left(t, S, E, A, I, Q, u_{1}\right)=M_{1} E(t)+M_{2} A(t)+ \\
& M_{3} I(t)+M_{4} Q+\frac{1}{2} k_{1} u_{1}^{2}(t)+\sum_{i=1}^{5} \lambda_{i}(t) g_{i}(t) \\
& =\left(M_{1} E+M_{2} A+M_{3} I+M_{4} Q\right)+\frac{1}{2}\left(k_{1} u_{1}^{2}\right)+ \\
& \lambda_{1}\left[\pi+\theta Q-\left(1-u_{1}\right) \frac{\beta}{N}(I+q A) S-\mu S\right]+
\end{aligned}
$$$$
\lambda_{2}\left[\left(1-u_{1}\right) \frac{\beta}{N}(I+q A) S-\eta E-\varphi E-\mu E\right]+
$$$$
\lambda_{3}[(1-P) \eta E-\gamma A-(\mu+\zeta) A]
$$

$+\lambda_{4}[P \eta E-\alpha I-(\mu+\zeta) I]+\lambda_{5}[\alpha I+\gamma A+$ $\varphi E-(\theta+\mu) Q]$

where $\lambda_{i}(t)$, for $i=1,2,3,4,5$ are the adjoint functions to be determined suitably. The form of the adjoint equations and transversality conditions are standard results from PMP. The adjoint (costate) equation is the negative value of the Hamiltonian function differentiated with respect to each costate variable. It is given by

$$
\left\{\begin{aligned}
\frac{d \lambda_{1}}{d t}=-\left(\frac{\partial H}{\partial S}\right)= & \left(1-u_{1}\right) \frac{\beta}{N}(I+q A)\left(\lambda_{1}-\lambda_{2}\right)+\mu \lambda_{1}, \\
\frac{d \lambda_{2}}{d t}=-\left(\frac{\partial H}{\partial E}\right)=- & M_{1}+(\eta+\varphi+\mu) \lambda_{2}-(1-p) \eta \lambda_{3}- \\
& p \eta \lambda_{4}-\varphi \lambda_{5}, \\
\frac{d \lambda_{3}}{d t}=-\left(\frac{\partial H}{\partial A}\right)= & -M_{2}+\left(1-u_{1}\right) \frac{\beta}{N} q S\left(\lambda_{1}-\lambda_{2}\right)+ \\
& (\gamma+\mu+\zeta) \lambda_{3}-\gamma \lambda_{5}, \\
\frac{d \lambda_{4}}{d t}=-\left(\frac{\partial H}{\partial I}\right)=- & (\alpha+\mu+\zeta) \lambda_{4}-\alpha \lambda_{5}, \\
\frac{d \lambda_{5}}{d t}=-\left(\frac{\partial H}{\partial R}\right)= & -M_{4}+\theta\left(\lambda_{5}-\lambda_{1}\right)+\mu \lambda_{5} .
\end{aligned}\right.
$$

With transversality conditions: $\lambda_{i}(T)=0$, for $i=1,2,3,4,5$.

Stationary condition is obtained by differentiating Hamiltonian function with respect to control variable $\left(u_{1}\right)$. By the optimality condition, we have: $\frac{\partial H}{\partial u_{1}}=0$.

$$
\begin{aligned}
& \text { (i) } \frac{\partial H}{\partial u_{1}}=k_{1} u_{1}+\frac{\beta(I+q A)}{N} S\left(\lambda_{1}-\lambda_{2}\right)=0 a t \\
& u_{1}=u_{1}^{*}(t) \\
& u_{1}^{*}(t)=\frac{\beta\left(I^{*}+q A^{*}\right) S^{*}\left(\lambda_{2}-\lambda_{1}\right)}{N k_{1}},
\end{aligned}
$$

Proof: The Hamiltonian function associated so that by using the bounds for the control $u_{1}(t)$, 
we obtain

$$
u_{1}^{*}(t)= \begin{cases}\frac{\beta\left(I^{*}+q A^{*}\right) S^{*}\left(\lambda_{2}-\lambda_{1}\right)}{N k_{1}} \text { if } 0 \leq \frac{\beta\left(I^{*}+q A^{*}\right) S^{*}\left(\lambda_{2}-\lambda_{1}\right)}{N k_{1}} \leq 1, & \begin{array}{l}
S(0)=3000, E(0)=7000, A(0)= \\
0, \text { if } \frac{\beta\left(I^{*}+q A^{*}\right) S^{*}\left(\lambda_{2}-\lambda_{1}\right)}{N k_{1}} \leq 0,
\end{array} \quad \begin{array}{l}
600, I(0)=500 \text { and } Q(0)=100 . \text { For the } \\
\text { adjoint system we set terminal conditions }
\end{array} \\
1, \text { if } \frac{\beta\left(I^{*}+q A^{*}\right) S^{*}\left(\lambda_{2}-\lambda_{1}\right)}{N k_{1}} \geq 1, & \text { (5.17) } \begin{array}{l}
\lambda(T)=0 ; i=1,2,4,5 \text { where } T=100 \text { is }
\end{array}\end{cases}
$$

In compact notation optimal control $u_{1}$ can be simplified: $u_{1}^{*}=\min \left\{\max \left\{0, \frac{\beta\left(I^{*}+q A^{*}\right) S^{*}\left(\lambda_{2}-\lambda_{1}\right)}{N k_{1}}\right\}, 1\right\} \begin{array}{r}\text { they are not } 100 \% \text { effective. The cost coefficients } \\ \text { corresponding to control variables are estimated }\end{array}$

simulation time. We considered the numerical value of the controls $u_{1}$; between zero and one as to be $k_{1}=2$ and the relative importance of reState equation is obtained by differentiating Hamiltonian function with respect to each costate variable. Using (5.18) we obtain the following optimality system: ducing the associated classes on the spread of the disease are $M_{1}=1, M_{2}=3, M_{3}=2, M_{4}=5$. Then the simulation process are performed using software MATLAB $R 2015 b$ with ode 45 solver

$$
\left\{\begin{array}{l}
\frac{d S}{d t}=\frac{\partial H}{\partial \lambda_{1}}=\pi+\theta Q-\left(1-\left[\min \left\{\max \left\{0, \frac{\beta\left(I^{*}+q A^{*}\right) S^{*}\left(\lambda_{2}-\lambda_{1}\right)}{N k_{1}}\right\}, 1\right\}\right]\right) \lambda S-\mu S, \\
\frac{d E}{d t}=\frac{\partial H}{\partial \lambda_{2}}=\left(1-\left[\min \left\{\max \left\{0, \frac{\beta\left(I^{*}+q A^{*}\right) S^{*}\left(\lambda_{2}-\lambda_{1}\right)}{N k_{1}}\right\}, 1\right\}\right]\right) \lambda S-(\mu+\eta+\varphi) E \\
\frac{d A}{d t}=\frac{\partial H}{\partial \lambda_{3}}=(1-p) \eta E-(\mu+\gamma+\zeta) A \\
\frac{d I}{d t}=\frac{\partial H}{\partial \lambda_{4}}=p \eta E-(\mu+\alpha+\zeta) I \\
\frac{d Q}{d t}=\frac{\partial H}{\partial \lambda_{5}}=(\alpha I+\gamma A+\varphi E+)-(\mu+\theta) Q
\end{array}\right.
$$

subject to the following initial conditions:

$S(0)=S_{0}, E(0)=E_{0}, A(0)=A_{0}$,

$I(0)=I_{0}, Q(0)=Q_{0}$, and

$\lambda_{1}(T)=0, \lambda_{2}(T)=0, \lambda_{3}(T)=0$,

$\lambda_{4}(T)=0, \lambda_{5}(T)=0$,

This completes the proof.

\section{NUMERICAL SIMULATION OF THESYSTEM}

In this section, the numerical solutions of optimality system are discussed. To conduct the study, a set of meaningful values are assigned to the model parameters. These values are either taken from literature or assumed. Using the parameter values given in table 2 below and the initial conditions
Table 2: The values of parameters used in the simulations

\begin{tabular}{|c|c|c|}
\hline \hline Parameter & Value & Reference \\
\hline$\pi$ & 2 & {$[2]$} \\
\hline$\beta$ & 0.064 & {$[2]$} \\
\hline$\eta$ & 0.0024 & {$[1]$} \\
\hline$\alpha$ & 0.016 & {$[12]$} \\
\hline$\varphi$ & 0.4 & assumed \\
\hline$\theta$ & 0.0002 & {$[17]$} \\
\hline$\gamma$ & 0.054 & assumed \\
\hline$q$ & 1.002 & assumed \\
\hline$p$ & 0.067 & assumed \\
\hline$\mu$ & 0.02 & {$[1]$} \\
\hline$\zeta$ & 0.001 & {$[17]$} \\
\hline
\end{tabular}



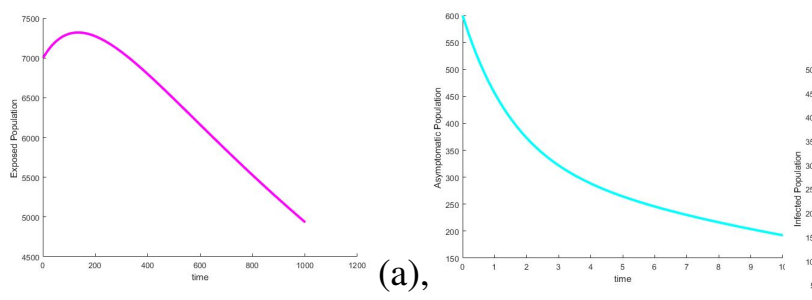

Figure 2: Dynamics of Exposed and Asymptomatic individuals without optimal control

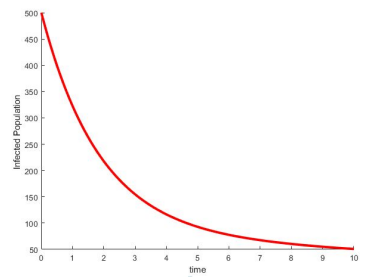

(c)

Figure 3: Dynamics of Infected and Quarantined individuals without optimal control

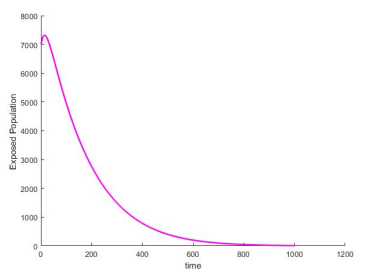

(e),

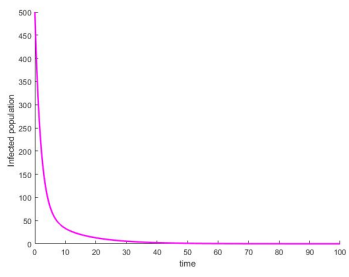

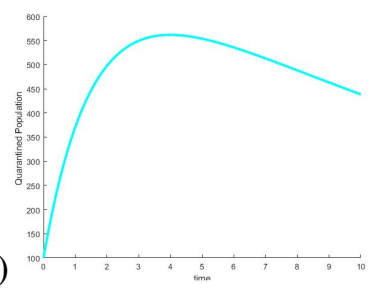

Figure 7: Dynamic of Infected and Quarantined individuals with and without optimal control
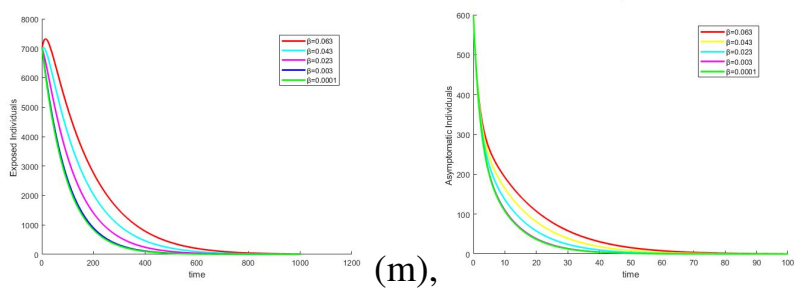

(n)

Figure 8: Dynamics of reducing effective contact rate with Exposed and Asymptomatic individuals by applying optimal control.

(f)

Figure 4: Dynamics of Exposed and Infected individuals with optimal control
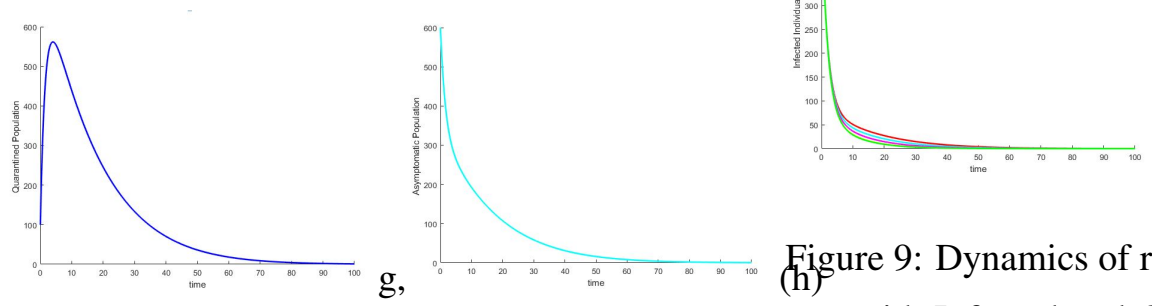

(o),

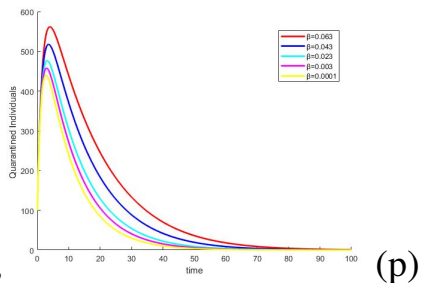

Figure 9: Dynamics of reducing effective contact rate with Infected and Quarantined individuals

Figure 5: Dynamics of Quarantined and Asymp- by applying optimal control. tomatic individuals with optimal control
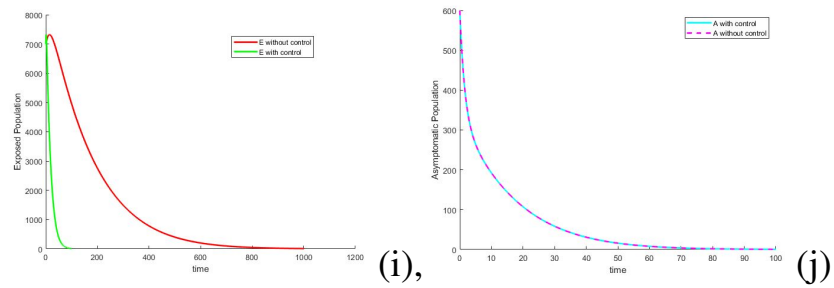

Figure 6: Dynamics of Exposed and Asymptomatic individuals with and without optimal control

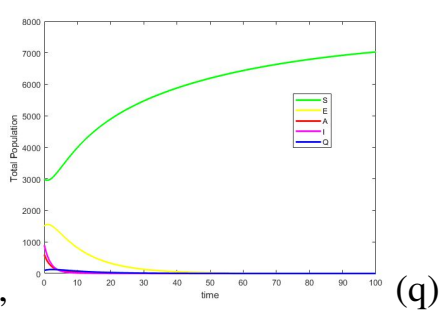

Figure 10: Total population with optimal control 
From the graph of numerical simulation , considering our assumption, initial condition and parameter values we deduce the following: Figure2(a), 2(b),3(c), 3(d) shows the dynamics of exposed, asymptomatic and infeccted individuals with out optimal control such that all exposed, asymptomatic and infected individuals are decreasing by joining the quarantined class due to reduced the transmission dynamics of the disease.Figure $3 d$ show that quarantined individuals increased firstly due to more number of exposed, asymptomatic and infected join the class and latr on decreased due to the reason that quarantined individuals joins the susceptible class. Figure $4(\mathrm{e}), 4 \mathrm{f}), 5 \mathrm{~g}), 5 \mathrm{~h})$ shows that the dynamics of exposed, infected, quarantined and asymptomatic individuals with optimal control respectively such that they damped down when control is applied. Figure 6(i) 6(j), 7(k), 7(1) shows that the dynmacis of exposed, asymptomatic, infected and quarantined individuals with and without optimal control at the same time such that infctious individuals damped down when control is applied and raised when control is not applied. Figure $8(\mathrm{~m})$, $8(\mathrm{n}), 9(\mathrm{o}), 9(\mathrm{p})$ shows that the dynamic of varying effective contact rate on exposed, asymptomatic, infected and quarantined individuals such that decreasing the effective contact rate with these population minimize the transmission dynamics of the disease. Figure 10(q) shows that the dynamics of the total population with optimal control such that applying the optimal control on the total population increased the susceptible individuals and decreased the exposed, asymptomatic, infeccted and quarantined individuals. In general from this simulation we have seen that that impact of control on susceptible, exposed, asymptomatic, infected and quarantined population respectively. It show that the density of susceptible population is increasing in the presence of the optimal control while the population of exposed, asymptomatic, infected and quarantined individuals are damped down when control is applied. Thus, as expected the number of susceptible populatin $S(t)$ is increasing in the presence of control and at the same time the number of exposed $E(t)$, asymptomatic $A(t)$, infected $I(t)$ and quarantined $Q(t)$ populations are decreasing in the presence of control and increasing in the absence of control.

\section{CONCLUSSION AND RECOMMENDATION}

In this paper, we have considered a special disease, COVID-19 infection. We have developed a five-compartmental model of COVID-19 infectious disease, namely: susceptible, exposed, asymptomatic, infective and quarantined populations and investigated the dynamical behavior of this model. With the next generation matrix method, we have found as basic reproduction number of the system, which helps us to determine the dynamical behavior of the system. We have established two distinct equilibriums for the model with both local and global stability on the disease-free and endemic equilibrium points. The disease free equilibrium point $E_{0}$ is local asymptotic stability when $R_{0}<1$. When $R_{0}>1$, the endemic equilibrium $E_{1}$ exists and the system becomes unstable at $E_{0}$ and locally asymptotically stable at $E_{1}$ under some conditions.

We setup an optimal control problem relative to the epidemic model so as to minimize the exposed, asymptomatic, infective and quarantined populations as well as to minimize the cost of control. We have considered the prevention controls as it reduce the transmission dynamic 
of COVID-19 infection. The control functions $u_{1}$ (preventation i.e (vaccination, using mask, hand washing, keeping social distance etc))are designed in such away that they minimize the objective functional (cost function) as given in (5.12).

It has been found that optimal control can more effectively reduce the number of exposed, asymptomatic infective and quarantined individuals by preventing the susceptible population. Controlling the spread of epidemics is currently a complex and important research topic. So, to predict and identify the cost-effective strategies to control the spread of COVID-19 and minimize the cost of the control programme are the primary goals of health administrators and policy-makers. Since eradication of COVID-19 infection remains a challenge we recommend that, the government should give a vaccination for susceptible individuals before the out breaking of the disease and introduce education programmers for the whole population. Also, there is need to increase the number of hospitals to deal with COVID-19 infection because COVID-19 infections pose serious health problem.

\section{Data Availability}

Data we used in this paper are available without any limitation.

\section{Conflicts of Interest}

The authors declare that they have no conflicts of interest.

\section{REFERENCES}

[1] Diekmann, O., Heesterbeek, J. A. P., and Metz, J. A. On the definition and the computation of the basic reproduction ratio $R_{0}$ in models for infectious diseases in heterogeneous populations. Journal of mathematical biology (1990).

[2] Castillo-Chavez, C., Feng, Z., and Huang, W. On the computation of $R_{0}$ and its role on Mathematical approaches for emerging and reemerging infectious diseases(2002).

[3] W. Ming, J. V. Huang, and C. J. P. Zhang, Breaking down of the healthcare system: mathematical modelling for controlling.

[4] A. Saltelli, K. Chan, and E. M. Scott, editors (2000). Sensitivity Analysis. Wiley Series in Probability and Statistics, Wiley.

[5] Website;https://en.wikipedia.org/wiki/Coronavirus/.

[6] Coronavirus: Common Symptoms, Preventive Measures, and How to Diagnose It; https: //www. caringlyyours.com/coronavirus/, Caringly Yours. Retrieved January 28, 2020

[7] E. D. Gurmua et al Mathematical Model of Novel COVID-19 and Its Transmission Dynamics

[8] World Health Organization Website. https://www.who.int/.

[9] Coronavirus: Common Symptoms, Preventive Measures, and How to Diagnose It; https: //www. caringlyyours.com/coronavirus/, Caringly Yours. Retrieved January 28, 2020

[10] J. Jia, J. Ding, S. Liu, G. Liao, J. Li, B. Duan, G. Wang and R. Zhang, Mod- 
eling the control of COVID-19: impact of policy interventions and meteorological factors, Electronic Journal of Differential Equations, 2020 (2020).

[11] T.-M. Chen , J. R., Q.-P. Wang, Z.-Y. Zhao, J.-A. Cui and L. Yin, A mathematical model for simulating the phase-based transmissibility of a novel coronavirus, Infectious Diseases of Poverty, 9 (2020).

[12] X. Rong, L. Yang, H. Chu and M. Fan, Effect of delay in diagnosis on transmission of COVID-19, MBE, (2020) .

[13] C. Yang and J. Wang, A mathematical model for the novel coronavirus epidemic in Wuhan, China, MB.(2020).

[14] Y. Li, B. Wang, R. Peng, C. Zhou, Y. Zhan, Z. Liu et al., Mathematical modeling and epidemic prediction of COVID-19 and its significance to epidemic prevention and control measures, Annals of Infectious Disease and Epidemiology (2020).

[15] La Salle, J. P. (1976). The stability of dynamical systems. Society for Industrial and Applied Mathematics.

[16] S. Edward and N. Nyerere, A mathematical model for the dynamics of cholera with control measures, Applied and Computational Mathematics (2015).

[17] Hamby, D. M. (1994). A review of techniques for parameter sensitivity analysis of environmental models. Environmental monitoring and assessment.

[18] Chitnis, N., Hyman, J. M., and Cushing, J. M. (2008). Determining important parameters in the spread of malaria through the sen- sitivity analysis of a mathematical model. Bulletin of mathematical biology.

[19] A. Gorbalenya, S. Baker, R. Baric, R. de Groot, C. Drosten, A. Gulyaeva, et al, The species Severe acute respiratory syndromerelated. coronavirus: classifying 2019-nCoV and naming it SARS-CoV-2, Nat. Microbiol (2020).

[20] Van den Driessche, P., and Watmough, J. (2002). Reproduction numbers and subthreshold endemic equilibria for compartmental models of disease transmission. Mathematical biosciences.

[21] Heesterbeek, J. A. P., and Dietz, K. (1996). The concept of $R_{0}$ in epidemic theory. Statistica Neerlandica.

[22] Abdulrahman, S., Akinwande, N. I., Awojoyogbe, O. B., and Abubakar, U. Y. (2013). Sensitivity analysis of the parameters of a mathematical model of hepatitis $b$ virus transmission. Universal Journal of Applied Mathematics

[23] Fleming, W. H., and Rishel, R. W. (2012). Deterministic and stochastic optimal control (Vol. 1). Springer Science and Business Media.

[24] Perelson, A. S., Essunger, P., Cao, Y., Vesanen, M., Hurley, A., Saksela, K., ... and Ho, D. D. (1997). Decay characteristics of HIV-1-infected compartments during combination therapy.

\section{Creative Commons Attribution License 4.0 (Attribution 4.0 International, CC BY 4.0)}

This article is published under the terms of the Creative Commons Attribution License 4.0 https://creativecommons.org/licenses/by/4.0/deed.en_US 